\title{
TEIA-USP - LABORATÓRIO DE EDUCAÇÃO E AMBIENTE / UNIVERSIDADE DE SÃO PAULO
}

\author{
Pedro Roberto Jacobi ${ }^{1}$
}

Resumo - O TEIA-USP - Laboratório de Educação e Ambiente foi criado em 2006 com o objetivo de estimular e promover um diálogo interdisciplinar que contribua para reflexões, debates, análises e construção de novos conhecimentos. Tem a proposta de realizar intervenções de caráter socioeducativo para uma prática reflexiva e engajada a partir da instituição acadêmica. Organiza-se por meio da agregação de docentes de cinco unidades da USP, de pesquisadores doutores e de alunos de pós-graduação num espaço cooperativo de pesquisa, reflexão e disseminação de conhecimentos sobre Educação Ambiental - teoria, prática e políticas. As suas atividades têm se concentrado na realização de seminários, palestras e grupos de estudos que incorporam os temas de pesquisa dos alunos envolvidos. O TEIA-USP tem um site cuja prioridade é facilitar o acesso à literatura sobre Educação Ambiental, temas transversais e abordagens teóricas da aprendizagem social e das dinâmicas cooperativas, além de divulgar publicações recentes e práticas educativas.

Palavras-chave: grupo de pesquisa, educação ambiental, pesquisa-ação.

Abstract - TEIA-USP - Education and Environment Laboratory was created in 2006 with the goal to stimulate and promote an interdisciplinary dialogue that contributes to reflections, debates, analysis and the formulation of new knowledge. It proposes socio-educational interventions to develop reflective and engaged practices through and within an academic institution. It is organized by the gathering of professors of five educational schools at USP, $\mathrm{PhD}$ researchers and graduate students within a cooperative space of research, analysis and dissemination of knowledge on Environmental Education - theory, practices and policies. Its activities have been concentrating on seminars, workshops, lectures and study groups that incorporate the themes researched by the graduate students involved. TEIA-USP has a website, and its priority is to facilitate the access to the literature on Environmental Education, transversal themes, and theoretical approaches to social learning and cooperative dynamics, as well as to provide information on new publications and educational practices.

Keywords: research group, environmental education, action research.

${ }^{1}$ Docente da Faculdade de Educação / USP e do Programa de Ciências Ambientais / USP. Contato: prjacobi@usp.br . 


\section{Apresentação}

O TEIA-USP - Laboratório de Educação e Ambiente foi criado em 2006 com o objetivo de estimular e promover um diálogo interdisciplinar que contribua para reflexões, debates, análises e construção de novos conhecimentos. Além disso, tem a proposta de realizar intervenções de caráter socioeducativo, para uma prática reflexiva e engajada a partir da instituição acadêmica. Organizase por meio da agregação de diversos pesquisadores num espaço cooperativo de pesquisa/reflexão/disseminação de conhecimentos sobre educação ambiental teoria, prática e políticas. Atualmente envolve docentes, pesquisadores e alunos de pós-graduação. As reuniões são mensais para estudos sobre teorias e práticas que envolvam Educação, Ambiente e Participação.

\section{Objetivos}

O desafio do grupo TEIA-USP é constituir um espaço interdisciplinar que possibilite uma relação qualificada com a atividade de pesquisa acadêmica e buscar a participação direta da comunidade acadêmica no debate e na elaboração de políticas públicas voltadas ao conjunto da sociedade, ao aproximar o conhecimento gerado junto aos organismos públicos e privados e comunidade em geral através de parcerias.

\section{Atividades}

O grupo tem desenvolvido diversos projetos de pesquisa de alcance nacional e internacional em nível de Mestrado e Doutorado. No ano de 2009, o TEIAUSP promoveu, no âmbito de extensão universitária, curso de curta duração (15 semanas) "Navegando nas águas do Pirajuçara" orientado para a formação de professores e apoio pedagógico, com atividades presenciais e à distância. Além disso, mensalmente realizamos seminários intitulados "Diálogos do TEIA" que, em 2010, desenvolveu o tema "Redes sociais, cidadania e sustentabilidade" sendo um espaço de interlocução entre a universidade e organizações não governamentais, órgãos públicos, sociedade civil e interessados em geral. Esse diálogo tem sido bastante auxiliado pelo nosso site www.teia.fe.usp.br, um espaço virtual para divulgação de eventos sobre Educação e Ambiente e que torna acessíveis diversas publicações, pesquisas, artigos e matérias produzidas pelo grupo de pesquisadores do TEIA-USP.

\section{Linhas de pesquisa}

- Governança da água e aprendizagem social

- Ecologia Marinha, Agenda 21 e educação comunitária

- Políticas públicas ambientais e educacionais

- Participação cidadã e protagonismo juvenil 
- Sustentabilidade, análise de discurso e mídia

- Arte-educação e educação ambiental crítica

- Geotecnologias e formação de professores

- Educação e Mudanças Climáticas

- Pedagogia da Educação Ambiental nas Organizações

\section{Problemas de pesquisa e objetivos gerais das investigações}

O campo da Educação Ambiental, na atualidade, tem enfatizado a necessária mudança de valores e atitudes, a adoção de referenciais éticos de solidariedade e valorização da vida; uma postura filosófica, epistemológica e pedagógica aberta à incerteza, à indefinição, à complexidade das relações na sociedade. Para essa transformação do potencial educativo na incorporação dos desafios e princípios da sustentabilidade, Pedro Roberto Jacobi ressalta a necessária mudança paradigmática, orientada por mudanças de percepção e de valores, capazes de conduzir a educação das gerações atuais rumo a novas leituras e interpretações da realidade socioambiental. As pesquisas desenvolvidas e em desenvolvimento tem se caracterizado pela diversidade, embora com um enfoque que privilegia as diferentes práticas socioeducativas.

Um dos temas que tem gerado uma lógica cooperativa no grupo foi a participação em atividades de ensino-pesquisa associadas com a elaboração da Agenda 21 na educação, que tem como premissa e condição, o envolvimento de uma gama de atores no processo de pensar e refletir sobre seu espaço de vida, os potenciais e desafios de seu patrimônio, de seu território de vida cotidiana. Abre caminhos para incrementar o potencial da escola que, embora atrelada ao institucional, pode se tornar um espaço possível de diálogos horizontalizados, de aprendizagem do exercício da democracia participativa, mediando experiências de diferentes sujeitos autores-atores locais na construção de projetos de intervenção coletivos. A prática concreta de investigação e ação, registro e reflexão sobre os processos e os resultados da implementação de um projeto de intervenção ambiental de caráter sócio-educativo, é embasado nos marcos conceituais da pedagogia crítica, da sociologia crítico-reflexiva, da psicologia sócio-histórica e da metodologia da pesquisa-ação participante.

Outro tema articulador é associado com as práticas que mobilizam atores sociais como é o caso dos jovens e seu engajamento no movimento ambiental. Neste sentido, coloca-se a necessidade de pensar em formas criativas de engajar os estudantes em práticas significativas. Providenciar meios para que possam criar comunidades de prática, ingressar nas que já existem e ampliar sua interação naquelas em que já participam são ações estratégicas para alargar o potencial de aprendizagem e de formação da identidade dos jovens. Uma estratégia que irá também enriquecer as próprias comunidades, um benefício tanto para o indivíduo quanto para o coletivo. Os grupos ambientalistas juvenis também atuam como espaços/estruturas educadoras. Esses grupos criam 
"comunidades aprendentes" que vivenciam os ensinamentos da educação popular, favorecem a prática do diálogo (processo dialógico), valorizam a experiência de vida de cada um (processo histórico) e desenvolvem a capacidade de problematizar a realidade e inspirar práticas individuais e coletivas orientadas para a transformação cultural (processo político e participativo).

Um terceiro foco de pesquisa é vinculado ao conceito de Aprendizagem Social e suas múltiplas possibilidades. A abordagem da "aprendizagem social' (Social Learning) parte da premissa de que os conflitos sócio-ambientais requerem não só o conhecimento técnico para o entendimento dos conteúdos envolvidos, mas também a incorporação da dimensão humana no processo de gestão. Essa aprendizagem envolve as interações dos diversos atores, a busca de uma visão compartilhada do problema e o processo de negociação dos diversos interesses, muitas vezes conflitantes no processo de gestão.

A ênfase das pesquisas recai sobre a análise das relações entre sujeitos e a construção de novos significados e novas subjetividades que contribuem para a apropriação de conhecimentos através da leitura crítica da realidade; e a possibilidade de constituição de um coletivo de sujeitos autores, identidades coletivas necessárias à construção de ações refletidas, engajadas e comprometidas em projetos de intervenção sobre a realidade socioambiental.

\section{Referenciais teórico-metodológicos}

Um dos importantes referenciais das pesquisas é Edgar Morin para quem o mito do progresso, a industrialização, a especialização, a fragmentação da ciência e da tecnologia e o individualismo, característicos das civilizações modernas, têm gerado conseqüências nas múltiplas dimensões da vida humana: solidão, egocentrismo, degradação da solidariedade, fragmentação de identidades. As ênfases são na práxis educativa na condição de pesquisadores e educadores com enfoque na cidadania ambiental no campo formal e não formal da educação, num contexto em que a identificação é como sujeito da pesquisa - sujeito aprendente e ensinante; que tem, portanto, uma concepção de mundo, de sujeito e subjetividade, de autor e autoria, de educação e de processo. As pesquisas têm-se orientado em muitos casos pela adoção de um modelo aberto de pesquisa qualitativa inspirada nas abordagens multireferencial de Jacques Ardoino, transversal, existencial de René Barbier, e antropopedagógica, integral e sistêmica de André Morin.

$\mathrm{Na}$ temática das práticas cooperativas, um dos referenciais é Etienne Wenger, que desenvolve o conceito de "Comunidades de Prática", e destaca o aprendizado como um fenômeno que reflete a natureza social do homem, no contexto de suas experiências de participação no mundo. Alerta para a lacuna do sistema educacional que, freqüentemente, articula o ensino como se o aprendizado fosse um processo individual e desconectado das demais experiências da vida. Aponta, assim, que é na perspectiva da valorização do caráter social do aprendizado e dos aspectos colaborativos envolvidos que as comunidades de prática podem ser analisadas como promotoras de aprendizagem, enfatizando 
que grande parte do dia a dia do indivíduo se dá dentro das mesmas. Instaura-se, assim, a importância de reconhecer e fortalecer comunidades de prática orientadas para a promoção da sustentabilidade. Comunidades que não podem ser impostas, mas sim imaginadas, identificadas, estimuladas, cultivadas e valorizadas. Destaca o aprendizado como um fenômeno que reflete a natureza social do homem, no contexto de suas experiências de participação no mundo.

Já o conceito de Aprendizagem Social remete à Psicologia, tendo sido o termo proposto por Albert Bandura para referir-se ao processo de desenvolvimento cognitivo (aprendizagem) dos indivíduos no contexto social. Atualmente o conceito perpassa diversas correntes das Ciências Sociais e pretende contribuir para explicitar os objetivos de todos os atores envolvidos no processo; alcançar melhores soluções (mais democráticas) para as questões sócio-ambientais e melhores maneiras de gerenciar os conflitos. Diversos autores têm desenvolvido pesquisas sobre a temática como é o caso de Arjen Wals, Robert Dyball, Paul Hart, Marc Craps, Jim Woodhil, Daniella Tilbury, Claudia Pahl-Wostl, entre outros. Os conceitos de aprendizado organizacional e aprendizagem social têm recebido diversas críticas pelo fato de ser uma abordagem demasiado instrumentalista e orientada ao desenvolvimento de técnicas de gestão; entretanto, se considerada conjuntamente à questão da construção social do conhecimento e da realidade, as reflexões sobre a aprendizagem social contribuem para pensar as inter-relações entre ciência, sociedade e ambiente. Suas ferramentas visam facilitar, por meio da formalização participativa, seguida de exploração de cenários, a construção de uma representação compartilhada do funcionamento das bacias vertentes, que envolva não somente as relações entre atores e recurso, mas também as interações entre atores. Nesse sentido, a construção de Plataforma de Múltiplos Grupos de Interesses, por exemplo, pode potencializar a intervenção, sem perder de vista o processo de democratização (participação para todos) e de emancipação e empoderamento dos atores; da resolução de conflitos e da gestão integrada de recursos.

\section{Integrantes}

Docentes: .Dr. Pedro Roberto Jacobi (Coordenador); Dra. Denise de la Corte Bacci; Dr. Alexandre Turra; Dr. Marcos Bernardino.

Pesquisadores: Maria Isabel Gonçalves Correia Franco; Luciana Ferreira da Silva; Paulo Marco C. Gonçalves; Vania Santos, Ana Cristina C. dos Anjos, Renata Ferraz; Vera Catalão

Orientandos: Virginia Talaveira Tristão; Samia Nascimento Sulaiman; Isis Morimoto; Marisa Castellano Tsai, Tiago Nepomuceno; Ana Cristina C. dos Anjos; Marcos Louzada e Angélica Maran.

\section{Instituições participantes}

Faculdade de Educação (FEUSP), Escola de Artes, Ciências e Humanidades (EACH), Instituto de Geociências (IGC), Instituto de Oceanografia (IO) e Programa de Pós-Graduação em Ciência Ambiental (PROCAM). 


\section{Projetos}

JACOBI, Pedro Roberto. (coord.); BACCI, Denise; FRANCO, Maria Isabel; SANTOS, Vania N.; SINISGALLI, Paulo, FRACALANZA, Ana Paula; FERRAZ, Renata. Aprendizagem Social e sua aplicação nas relações entre Ciência e a Governança Ambiental: 4 Estudos de caso no Estado de São Paulo. Projeto CNPq- Edital Universal iniciado em 2010.

JACOBI, Pedro Roberto. (coord.); BACCI, Denise; FRANCO, Maria Isabel; SANTOS, Vania N.; SINISGALLI, Paulo; FRACALANZA, Ana Paula; FERRAZ, Renata; SULEIMAN, Samia. Aprendizagem social como ferramenta para educação ambiental - metodologias participativas e práticas cooperativas na gestão compartilhada dos recursos hídricos na sub-bacia hidrográfica Cotia-Guarapiranga. Projeto Fehidro iniciado em 2011.

JACOBI, Pedro Roberto. (coord.); BACCI, Denise; FRANCO, Maria Isabel; SANTOS, Vania N.; NOVAES, Ricardo. Diagnóstico socioambiental, aprendizagem social e modelos multi-agentes na definição de políticas públicas para a gestão integrada dos recursos hídricos e uso e ocupação do solo. Projeto Fapesp Politicas Públicas. Concluído em maio 2011.

JACOBI, Pedro Roberto. (coord.); BEDUSCHI, Luiz C.; BACCI, D.; MORETO, E., NOVAES, R.; SINISGALLI, P. Aprendizagem Social na Gestão Compartilhada de Recursos Hídricos. Projeto CNPq- Edital Universal. Projeto concluído em 2010.

NOVAES, Ricardo. Educação Ambiental nos Projetos Fehidro em São Paulo- RMSP. Avaliação de Experiências. Pos Doc. FAPESP. Projeto concluído em 2010.

PATACA, Ermelinda. Formação inicial e continuada de professores em geociências e Educação Ambiental. FEUSP/LIGEA/USP

BACCI, Denise de la Corte. Avaliação dos Conceitos de senso comum em Geociências de alunos e professores do ensino fundamental e médio. IGc/LIGEA-USP.

PATACA, E.; Bacci, Denise. Geotecnologias como apoio à elaboração de material didático para o ensino fundamental: Atlas Ambiental Escolar da Região Metropolitana de Campinas. Embrapa.

SANTOS, Vania. Educação Ambiental Escolar e Realidade Socioambiental local:contribuições para a formação de professores e exercício da cidadania. Pós Doc. CAPES. Concluído em 2010. 


\section{Teses e dissertações defendidas}

ANJOS, Ana Cristina C. dos. Arte-Educação e Educação Ambiental. Uma reflexão sobre a colaboração teórica e metodológica da Arte-Educação para a Educação Ambiental. Orientador: Maria Christina de Souza-Lima Rizzi. São Paulo, 2010. Dissertação (Mestrado - Programa de Pós-Graduação em Artes. Área de Concentração: Arte-Educação) - Escola de Artes e Comunicação da Universidade de São Paulo.

FRANCO, Maria Isabel Gonçalves Correia. Educação Ambiental e Pesquisa-Ação Participante:registro analítico-crítico de uma prática educativa. Orientação: Pedro Roberto Jacobi. São Paulo:s.n., 2010. 212 p. : il + CDRom. Tese de Doutorado. Área de Concentração: Cultura, Organização e Educação - Faculdade de Educação da Universidade de São Paulo.

GONÇALVES, Paulo Marco de Campos. Anticorpos de Gaia no encontro das águas: trajetórias de aprendizagem de jovens nas trilhas do ambientalismo. Orientação Pedro Roberto Jacobi. São Paulo: s.n., 2010. 272 p. : il. ; tab; anexos; apêndices. Tese (Doutorado - Programa de Pós-Graduação em Educação. Área de Concentração : Cultura, Organização e Educação) - Faculdade de Educação da Universidade de São Paulo.

SILVA, Luciana Ferreira da. Educação ambiental crítica: entre ecoar e recriar. Orientação Pedro Roberto Jacobi. São Paulo: s.n., 2009. 197 p. Tese (Doutorado Programa de Pós-Graduação em Educação. Área de Concentração : Cultura, Organização e Educação) - Faculdade de Educação da Universidade de São Paulo.

SULAIMAN, Samia Nascimento. Educação ambiental à luz da análise do discurso da sustentabilidade : do conhecimento científico à formação cidadã. Orientação: Pedro Roberto Jacobi. São Paulo: s.n., 2010. 132 p. : il. + 1 CDRom. Dissertação (Mestrado - Programa de Pós-Graduação em Educação. Área de Concentração : Cultura, Organização e Educação) - Faculdade de Educação da Universidade de São Paulo.

\section{Publicações}

BACCI, Denise de La Corte ; PATACA, Ermelinda. M. "Educação para a água". Estudos Avançados, v. 22, p. 211-226, 2008.

CAMARGO, M. E. ; JACOBI, Pedro Roberto . Jogos de Papéis em Diálogo com a Educação Ambiental: Aprendendo a Participar da Gestão de Recursos Hídricos na Região Metropolitana de São Paulo. In: RIBEIRO, Wagner Costa. (Org.). Rumo ao Pensamento Crítico Socioambiental. 328 ed. São Paulo: Annablume Editora, 2010, v.01, p. -309 .

FRANCO, Maria Isabel G. C. A abordagem territorial, subjetividades, identidades coletivas e sustentabilidade - implicações para a prática da pesquisa participante em educação ambiental". In Jacobi, P. R.; Tristão, M. (orgs.). Educação ambiental e os movimentos de um campo de pesquisa. São Paulo: Annablume, 2010. 264 p. 
GUERRA, Antonio Fernando S.; JACOBI, Pedro Roberto; SULAIMAN, Samia nascimento; NEPOMUCENO, Tiago Costa. Mudanças climáticas, mudanças globais: desafios para a educação. Revista Eletrônica do Mestrado em Educação Ambiental (REMEA). V. especial. Outubro, 2010. pp. 88-105. Disponível em: <http://www.remea.furg.br/edicoes/vesp2010/art7vesp2010.pdf>.

JACOBI, Pedro Roberto. "Meio Ambiente, Educação e Cidadania: diálogo desaberes e transformação das práticas educativas. In: ”JACOBI, Pedro R.; MONTEIRO, Fernando; FERNANDES, Lidia. Educação e Sustentabilidade: Caminhos e Práticas para uma Educação Transformadora. Evoluir Cultural, São Paulo, 2009.108p.

JACOBI, Pedro Roberto, TRISTÃO, Martha. (orgs) Educação Ambiental e os movimentos de um campo de pesquisa. São Paulo, Annablume, 2010. 264p.

JACOBI, Pedro Roberto. Co-autoria com RIBEIRO, H; BESEN, G.R.; GUNTHER, W.; DEMAJOROVIC, J.; Viveiros, M. Coleta Seletiva com Inclusão Social. Annablume, São Paulo, 2009. 111p.

JACOBI, Pedro Roberto; GUERRA, Antonio F. S.; SULAIMAN, Samia N.; NEPOMUCENO, Tiago. Mudanças climáticas globais: a resposta da educação. Sessão especial "Educação e mudanças globais" - Grupo de Trabalho $\mathbf{n}^{\circ} 22$ de Educação Ambiental, 33 ${ }^{a}$ Reunião da Associação Nacional de Pesquisa em Educação (ANPED). Outubro/2010.

JACOBI, Pedro Roberto; SULAIMAN, Samia N.; SILVA, Luciana Ferreira da; RATINHO, Lesly; NEPOMUCENO, Tiago. Education and Climate Change in Brazil Report for the IALEI Project - Climate change and sustainable development: The response from Education in Brazil. In: Climate Change and Sustainable Development: The Response from Education - CROSS-NATIONAL REPORT. Internacional Alliance Leadership Educational Institute - IALEI Project: Climate Change and Sustainable Development: The Response from Education, 2009.

JACOBI, Pedro Roberto; TRISTÃO, Martha; FRANCO, Isabel. A Função Social da Educação Ambiental nas Práticas Colaborativas: Participação e Engajamento. Cad. CEDES, Abr 2009, vol.29, nº.77, p.63-79.

SANTOS, Vânia M.N. Projetos escolares com imagens de satélite: ferramentas de pesquisa-ensino para o estudo do ambiente. PENTEADO, H.D; GARRIDO, E. (orgs). Pesquisa-ensino: a comunicação escolar na formação do professor. São Paulo: Editora Paulinas, 2010 (Coleção Educação em Foco).

SANTOS, Vânia M.N.; COMPIANI, Maurício. Formação de professores para o estudo do ambiente: projetos escolares e a realidade socioambiental local. Revista Terra e Didática: Geosciences-Geography-Environment. 2009. v.5, n.1, p. 72-86.

SANTOS, Vânia M.N.; JACOBI, Pedro Roberto. Formação de professores e cidadania: projetos escolares no estudo do ambiente. artigo aceito para publicação na Revista Educação e Pesquisa, da Faculdade de Educação da USP. 
SANTOS, Vânia M.N.; JACOBI, Pedro Roberto. Formação de professores para o estudo do ambiente e: realidade socioambiental local e cidadania. TRISTÃO, M.; JACOBI, Pedro Roberto (orgs). Educação ambiental e os movimentos de um campo de pesquisa. São Paulo: Annablume Editora, 2010.

SILVA, Luciana Ferreira da. Educação Ambiental Crítica, Movimento Social e Mudança do Clima. Pesquisa em Debate, São Paulo. 2004, v. 5-n1, p. 1-15, 2008.

SILVA, Luciana Ferreira da. Contribuição para o entendimento da criticidade da Educação Ambiental: Redes, Sociedade e Informática - legados não-neutros da modernidade. Pesquisa em Debate, São Paulo. 2004, v. 5 n2, p. 1-15, 2008.

SILVA, Luciana Ferreira da. Educação Ambiental Crítica e Gestão Escolar. Pesquisa em Debate, São Paulo. 2004, v. 06 n 1, p. 1-15, 2009.

SILVA, Luciana Ferreira da . Reflexões sobre Interdisciplinaridade e Educação Ambiental Crítica. Pesquisa em Debate, São Paulo. 2004, v. 6 n2, p. 1-15, 2009.

SILVA, Luciana Ferreira. Educação Ambiental Crítica e Trabalho - Entre Reproduzir e Produzir Criticamente. Anais..., São Carlos. V EPEA - Configurações do Campo de Pesquisa em Educação Ambiental. São Paulo : UFSCAR, 2009. p. 950-964

SILVA, Luciana Ferreira da. As Relações Entre Conhecimento Científico E Educação Ambiental Crítica Contribuição Metodológica Das Sociologias Das Ausências, Das Emergências E Tradução. In: JACOBI, Pedro Roberto; TRISTÃO, Martha. (orgs.). Educação ambiental e os movimentos de um campo de pesquisa. São Paulo: Annablume, 2010, p. 223-9.

SULAIMAN, Samia N. Governança da água no Chile: a contribuição do Sistema Nacional de Certificação Ambiental de Estabelecimentos Educacionais (SNCAE). Pesquisa em Debate, v.12, p.10 - 21, 2010.

SULAIMAN, Samia N. Educação ambiental, sustentabilidade e ciência: o papel da mídia na difusão de conhecimentos científicos. (Artigo aceito para publicação na Revista Ciência e Educação - UNESP, 2010).

SULAIMAN, Samia N.; TRISTÂO, Virgínia Talaveira Valentini. Estudo do Meio: uma contribuição metodológica à Educação Ambiental. Revista Eletrônica do Mestrado em Educação Ambiental (REMEA), v.21, p.341-355, 2008.

SULAIMAN, Samia N.; TRISTÃO, Virgínia Talaveira Valentini. Educação ambiental e sociologia ambiental: Interlocuções com a teoria da reflexividade. Pesquisa em Debate, v.6, p.1 - 15, 2009.

SULAIMAN, Samia N.; TRISTÃO, Virginia Talaveira Valentini; JACOBI, Pedro R. Educación Ambiental en la educación de Brasil: avances legales, políticas y educativas para su inclusión en la educación nacional. ANAIS do II Congreso nacional de Investigación en Educación Ambiental para la Sustentabilidad (CNIEAS). Puebla, México, 23-26 de março de 2011. 
TRISTÃO, José Américo Martelli; TRISTÃO, Virgínia Talaveira Valentini. Responsabilidade Corporativa: o projeto "Do Lixo às Flores". Pesquisa em Debate, São Paulo. 2004, v. 5 (2), p. 1-20, 2008.

TRISTÃO, Martha; JACOBI, Pedro Roberto . A Educação Ambiental e os Movimentos de um Campo de Pesquisa: Entre, Através e Além do Ambientalismo e da Educação. Educação Ambiental e os Movimentos de um Campo de Pesquisa. São Paulo: Annablume, 2010, p. 13-29.

TRISTÃO, Virgínia Talaveira Valentini. Educação Ambiental Não Formal em Parques Urbanos. Pesquisa em Debate, São Paulo, 2004, v. 5(1), p. 1-15, 2008.

TRISTÃO, Virgínia Talaveira Valentini . A educação ambiental nas trilhas do Jardim da Luz. Anais... São Paulo. V Fórum de Debates - Perspectivas Interdisciplinares nas Artes e nas Humanidades, 2007.

TRISTÃO, Virgínia Talaveira Valentini, CORTEZ, Eduardo P., MEDEIROS, Marcos, SILVA, Mariano R., TOLEDO, Michele, AMARAL, Sandra C., SHAHINI, Alexandre, L. Trilhas Interpretativas em Educação Ambiental: A Experiência do Programa Trilhas Urbanas no Parque Alfredo Volpi. In: $3^{\circ}$ Seminário de Parques e Áreas Verdes, 2010, São Paulo. $3^{\circ}$ Seminário de Parques e Áreas Verdes. Biodiversidade e Sustentabilidade: Experiências, Planos e Ações, 2010.

TRISTÃO, Virgínia Talaveira Valentini; JACOBI, Pedro R.. Educação ambiental empresarial: Parcerias entre empresas e ONGs. Anais..., 2010, São Paulo. XII Encontro Internacional sobre Gestão Empresarial e Meio Ambiente- ENGEMA, 2010.

TRISTÃO, Virgínia Talaveira Valentini; SULAIMAN, Samia Nascimento. Estudo do meio: uma leitura interdisciplinar e coletiva do espaço para o exercício da educação ambiental. Anais... São Paulo. III Seminário Internacional de Educação. Pesquisas em Educação no Brasil e no Mundo, 2010.

Artigo recebido em 20/08/2010 - $\quad$ aprovado em 17/12/2010 